\title{
Fibroblast growth factor 21 prolongs lifespan and improves stress tolerance in the silkworm, Bombyx mori
}

\author{
Jiang-Bo Song", Kai-Ge Hao", Xin Chen, Yan-Hua Zhang, Zi-Lin Cheng, Shuang Mao, Yong-Xi Tang, \\ Xiao-Ling Tong, Fang-Yin Dai
}

State Key Laboratory of Silkworm Genome Biology, Key Laboratory for Sericulture Biology and Genetic Breeding, Ministry of Agriculture and Rural Affairs, College of Biotechnology, Southwest University, Chongqing 400715, China

Contributions: (I) Conception and design: FY Dai, XL Tong; (II) Administrative support: FY Dai, XL Tong; (III) Provision of study materials or patients: FY Dai; (IV) Collection and assembly of data: JB Song, KG Hao, YX Tang, ZL Cheng, S Mao; (V) Data analysis and interpretation: JB Song, KG Hao, X Chen, YH Zhang, ZL Cheng, S Mao; (VI) Manuscript writing: All authors; (VII) Final approval of manuscript: All authors.

\#These authors contributed equally to this work.

Correspondence to: Fang-Yin Dai. State Key Laboratory of Silkworm Genome Biology, Southwest University, Tiansheng road No. 2, Beibei district, Chongqing 400715, China. Email: fydai@swu.edu.cn.

\begin{abstract}
Background: Fibroblast growth factor 21 (FGF21), an FGF family member, is an atypical hormone and pro-longevity factor.

Methods: To better understand of the effects of exogenous administration of FGF21 on lifespan and stress tolerance, and the underlying molecular basis, we used the silkworm, Bombyx mori, as an experimental animal model to evaluate FGF21's pharmaceutical effects.

Results: Lifespan was significantly prolonged in female silkworms with FGF21 replenishment, whereas no effect was observed in the male silkworms. FGF21 replenishment also significantly improved the activity of antioxidant systems such as glutathione-S-transferase (GST) and superoxide dismutase (SOD) and significantly decreased malondialdehyde (MDA) content. Moreover, FGF21 was found to play a critical role in enhancing stress resistance, including ultraviolet (UV) irradiation tolerance and thermotolerance. Furthermore, $A M P K, F_{0 x} O$, and sirtuins were activated by FGF21 and may be responsible for the prolonged lifespan and enhanced antioxidant activity observed in silkworms.

Conclusions: Collectively, the results suggest the molecular pathways underlying of FGF21-induced longevity and stress tolerance, and support the use of silkworms as a promising experimental animal model for evaluating the pharmaceutical effects of small molecules.
\end{abstract}

Keywords: Bombyx mori; fibroblast growth factor 21 (FGF21); lifespan; oxidation resistance; stress tolerance

Submitted Nov 19, 2019. Accepted for publication Dec 27, 2019.

doi: 10.21037/atm.2020.01.18

View this article at: http://dx.doi.org/10.21037/atm.2020.01.18

\section{Introduction}

Fibroblast growth factor (FGF) is excreted by smooth muscle cells, endothelial cells, and macrophages (1). The FGF subfamily can be classified into the following subfamilies on the basis of sequence similarity, biochemical function and evolutionary relationships: one subfamily of endocrine FGFs, one subfamily of intracellular FGFs, and five subfamilies of paracrine FGFs (2). To date, FGF1$F G F 23$ genes have been identified in many species, including humans and mice (3).

The FGF15/19 subfamily belongs to the subfamily of endocrine FGFs, including FGF15/19, FGF21, and FGF23, and plays critical roles in body homeostasis maintenance, glucose regulation, insulin sensitivity, and lipid metabolism, via Klotho dependence (4). FGF21, a hormone-like member of the FGF family, coordinates interactions and is a potent longevity factor linking energy metabolism and stress responses (5). Many studies have shown that FGF21 
treatment can mitigate some age-related metabolic disorders and cardiovascular diseases. Additionally, transgenic mice overexpressing FGF21 have prolonged lifespans (6).

Recent studies have revealed that certain molecular mechanisms through FGF21 signaling may be targets for interventions to increase the healthy lifespan (4). FGF21 activates $\mathrm{Nrf2}$ and suppresses the NF- $\mathrm{\kappa B}$ signaling, thus promoting macrophage-mediated inhibition of inflammation (7). Moreover, substantial evidence indicates that stress resistance capacity can generally enhance longevity across species $(5,8)$. Sirtuins, originally identified in Saccharomyces cerevisiae, are a conserved protein family named after the first member silent information regulator 2 (Sir 2) protein (2). Several studies have demonstrated that the activation of sirtuins may plausibly explain most of the geroprotective benefits observed from several perspectives in reports published to date (9). Activation of antioxidative enzyme systems through the actions of Rhodiola rosea, metformin, and Astragalus polysaccharide has been reported to extend the lifespan $(10,11)$. Herein, we detected the molecular basis underlying how FGF21 induces longevity through promoting interactions between stress responses and lifespan extension.

However, whether the role of FGF21 has a beneficial effect in scavenging free radicals and regulating resistance to oxidative damage remains unknown, as do the molecular underpinnings of FGF21 in longevity. Therefore, we examined antioxidative signaling pathways to elucidate the role of FGF21 in longevity. The conservative longevityrelated signaling pathway in diverse species principally includes four signaling pathways, namely, insulin/insulinlike growth factor 1 (IGF1), AMP activated protein kinase (AMPK), target of rapamycin (TOR) and sirtuin signaling pathway (9). Normally, AKT, transcription factor fork head box $\mathrm{O}$ (FoxO), AMPK, Tor1 and Tor2, and sirtuins were employed as representative genes in these four pathways.

Silkworms, because of their clear genetic background, excellent bioinformatics utility, and clear boundaries between developmental stages, are an advantageous model organism for research on aging, lifespan, and the effects of drugs on lifespan $(10,12)$. The high reproductive rate of silkworms can provide abundant larvae populations with the same developmental status and genetic background, thus enabling low cost determination of survival statistics (12). In addition, the silkworm is a non-sentient animal, thus presenting few ethical issues in experiments, and their size is conducive to observation of morphology and behavior, and to exogenous injection. Moreover, the low mobility of the larvae and the flightlessness of the moths enable environmentally friendly, convenient breeding, management, and lifespan measurement $(11,12)$. In addition, the open blood circulation system is highly sensitive to exogenous drugs, which spread throughout the body in only a few minutes (12). The standardized breeding of silkworms enables the reliability and repeatability of measured effects of drugs on the lifespan of silkworms without interference from diet and culture conditions (12). These characteristics have received the attention of researchers and provide substantial advantages in studies of silkworm aging and lifespan. Therefore, the silkworm was used as a model in the experiments in this study.

\section{Methods}

\section{Silkworm strain}

We used Dazao, a wildtype silkworm strain in the Silkworm Gene Bank at Southwest University. Silkworms were maintained under a relative humidity of approximately $75 \%$ to $50 \%$ at $25 \pm 0.5{ }^{\circ} \mathrm{C}$ with circadian rhythms of $12 \mathrm{~h}$ light/12 h dark cycle throughout the life cycle. Silkworms in the larval stage were reared with fresh mulberry leaves ad libitum $(10,11,13)$. All of the above procedures were consistent with the statement of Southwest University regarding animal welfare.

\section{FGF21 replenishment}

Commercial preparations of FGF21 were obtained from Sino Biological (Beijing, China). A $25 \mathrm{ng} / \mu \mathrm{L}$ stock solution of FGF21 was prepared in sterile deionized $\mathrm{H}_{2} \mathrm{O}$. After sterilization by filtration through $0.22 \mu \mathrm{m}$ membranes and storage at $4{ }^{\circ} \mathrm{C}$, the stock solution was injected with 5 or $10 \mu \mathrm{L}$ of $25 \mathrm{ng} / \mu \mathrm{L}$ FGF2 1 stock solution was injected per silkworm moth (treatment group), and deionized water was injected for the control group.

\section{Lifespan assays}

Throughout the life cycle, survival statistics were used to determine the lifespan expectancy and mortality rates of silkworms in an incubator with a suitable growth environment, as previously described $(10,11,13)$. The maximum lifespan was defined as the upper $10 \%$ of the lifespan distribution $(10,11,13)$. The survival conditions of the silkworm were determined on the basis of artificial 
Table 1 The primer sequences used in this study for quantitative real-time PCR

\begin{tabular}{|c|c|c|}
\hline Primer name & F primer $\left(5^{\prime}-3^{\prime}\right)$ & R primer (5'-3') \\
\hline$B m A M P K$ & GCACCCCTGTCAAACGAGC & CGTTCGCCCGACAAAGACT \\
\hline$B m A K T$ & GCTGCTAGACAAAGACGGACAC & ATGATGAGCCCGAACAGCAC \\
\hline BmTor1 & GCCACCTCCAAGCTACCCTAATAATGTT & AATCTATCCTTGCTTGTGTCGTGTTTC \\
\hline BmFoxO & GCACAGGACAACAGGCTCACAC & GCTTGGCGTCGGGATTGA \\
\hline BmSirt2 & TGGTCCCAGATTCGTGTCCT & TGTTCACGGGCTACTAATGCTC \\
\hline BmSirt4 & AGTTCAATGTCTCAAATGCCCC & CССТTCACACTTTGGGCAGA \\
\hline BmSirt5 & GCAAATAGAGGAGCCCCAGA & AAACTTTCACCGAACCACACAA \\
\hline BmHSP70 & GAAGGCACTCAAGGATGCTAAAC & TCAATACCGAGGGACAGAGGAG \\
\hline BmHSP90 & TCCCAGTTCATTGGCTACCC & TTGACTGCAAGATGGTCCTCC \\
\hline
\end{tabular}

identification, and the time of death was recorded every $3 \mathrm{~h}$.

\section{UV irradiation- and thermo-tolerance assays}

For stress induction, silkworm specimens were either incubated at $37^{\circ} \mathrm{C}$ for $12 \mathrm{~h}$ or subjected to ultraviolet (UV) exposure at $1.78 \mathrm{mw} / \mathrm{cm}^{2}$ for $12 \mathrm{~h}$ in the incubator. During the environment stress period, touch-provoked movement was used to determine survival, and the time of death was recorded.

Superoxide dismutase (SOD), glutathione-S-transferase (GST), catalase (CAT), and malondialdebyde (MDA) assays

To evaluate the drug effects on antioxidative activity in a given time span, we determined the activity of SOD and GST and CAT and the content of MDA in adults 1 day after FGF21 replenishment. SOD, GST, CAT, and MDA assay kits were used to measure whole-body homogenates according to the kit instructions.

\section{Reverse transcription-quantitative PCR (RT-qPCR)}

Silkworm specimens were collected from the treatment and control groups 24 and $48 \mathrm{~h}$ after FGF21 injection. Total RNA from three individuals was isolated with a rapid extraction Total RNA Kit (BioTeke Corporation, Beijing) in accordance with the manufacturer's instructions. RT-qPCR was performed with a CFX96 RealTime System (Bio-Rad, USA). Eukaryotic translation initiation factor 4A (BmMDB probe ID sw22934) served as a reference silkworm gene, owing to its optimum stability (14). Primer pairs targeting regions of every analyzed gene are shown in Table 1, and the relative expression levels of each gene were normalized to that of sw22934 and calculated as $2^{-\Delta \Delta C \mathrm{Ct}}$.

\section{Statistical analysis}

Statistical analyses were performed in GraphPad Prism 6 (GraphPad Software, CA, USA) and Excel (Microsoft, WA, USA). The maximum lifespan and average lifespan were calculated and reported as mean \pm SEM from independent experiments, and the significance of the differences was analyzed with two-tailed Student's $t$-test and two-way ANOVA. The significant differences were assessed with the log-rank test (Mantel-Cox) (PRISM software package, GraphPad software) in the survival curves. Values of $\mathrm{P}<0.05$ were considered statistically significant.

\section{Results}

\section{FGF21 promotes lifespan extension in female silkworms}

To reveal the effect of FGF21 on lifespan, we examined 
A

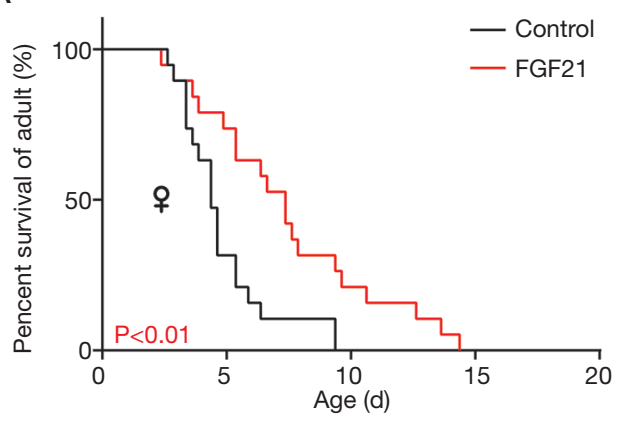

C

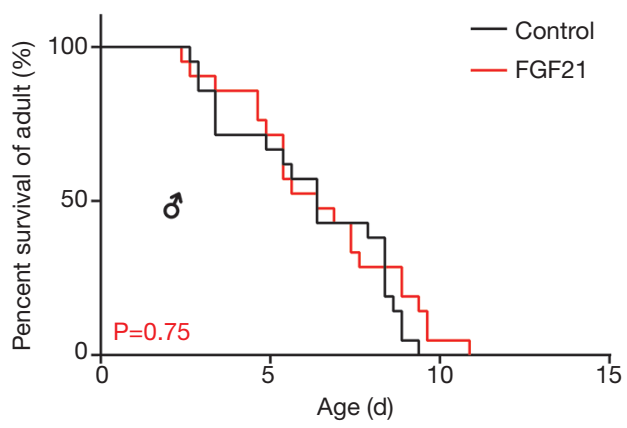

B

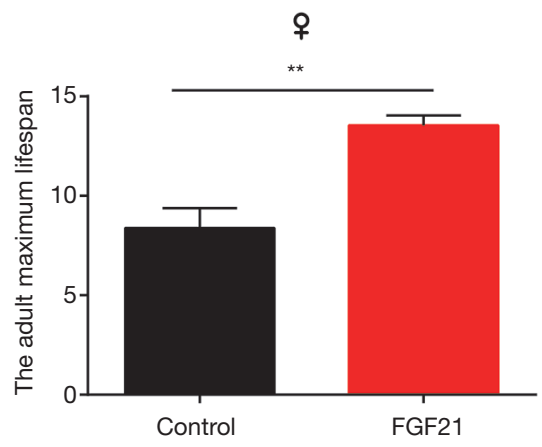

D

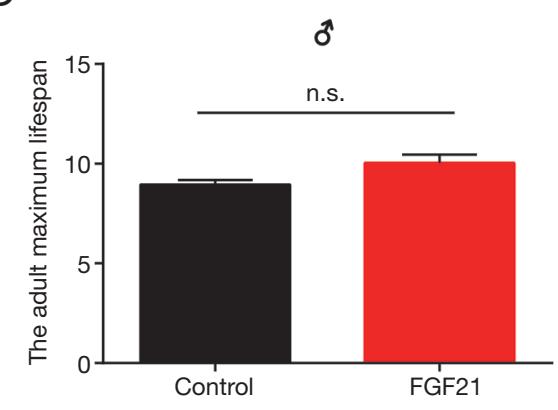

Figure 1 The effects of FGF21 on lifespan in silkworms. (A) The survival curve of unmated female silkworms, treatment ( $\mathrm{n}=20$ ) and control $(n=20)$; (B) the maximum lifespan in unmated female silkworms, treatment $(n=3)$ and control $(n=3)$; (C) the lifespan in unmated male silkworms, treatment ( $\mathrm{n}=31)$ and control $(\mathrm{n}=31)$; (D) the maximum number of days of unmated male silkworms, treatment ( $=3$ ) and control $(\mathrm{n}=3)$. Error bars depict the mean \pm SEM. ${ }^{* *}, \mathrm{P}<0.01$. FGF21, fibroblast growth factor 21; SEM, standard error of the mean.

silkworms fed mulberry leaves. The female silkworms lived longer with FGF21 replenishment than with the control treatment (Figure 1A,B); the mean lifespan in the adult stage was prolonged $54.42 \%$ (Figure $1 A$ ); and the maximum lifespan of the adult stage was also extended $61.69 \%$ (Figure 1B). There was no significant difference in the mean lifespan and maximum lifespan in the adult stage in male silkworms (Figure 1C,D). Thus, sex-specific regulation might possibly be involved in the process of FGF21induced lifespan extension.

\section{FGF21 protects silkworms from environmental stress}

Many recent studies have demonstrated that increased stress resistance can lengthen lifespan in multiple species (15-17). FGF21, as a stress hormone, has clear functions at the organism level in the maintenance of tissue homeostasis $(18,19)$. Thus, we hypothesized that FGF21 might play a role in protecting silkworms from environmental stress. To determine whether FGF21 could exert effects in response to environmental stress, we bred FGF21injected and control silkworms under thermal stress or UV irradiation. The thermotolerance was significantly improved with injection of $5 \mu \mathrm{L}$ (Figure $2 A$ ) or $10 \mu \mathrm{L}$ (Figure $2 B$ ) of FGF21 in silkworms, thus supporting survival. However, the expression differences of heat shock protein 70 (HSP70) and HSP90 were not detected in female and male silkworm with FGF21 replenishment after $12 \mathrm{~h}$ thermal stimulus compared counterpart (Figure 2C,D). However, the resistance of silkworms to UV irradiation did not significantly improve with injection of $5 \mu \mathrm{L}$ FGF2 1 (Figure 2E), but did significantly improve with injection of $10 \mu \mathrm{L}$ FGF21 (Figure $2 F$ ). Following, the antioxidant enzymes were detected with injection of $10 \mu \mathrm{L}$ FGF2 1 and the result showed that the activity of GST and SOD have no difference in female and male silkworm with FGF21 replenishment after UV stimulus compared control (Figure 2G,H), while the activity of catalase was increased and the contend of MDA was decreased in female and male with FGF21 replenishment after UV stimulus compared 

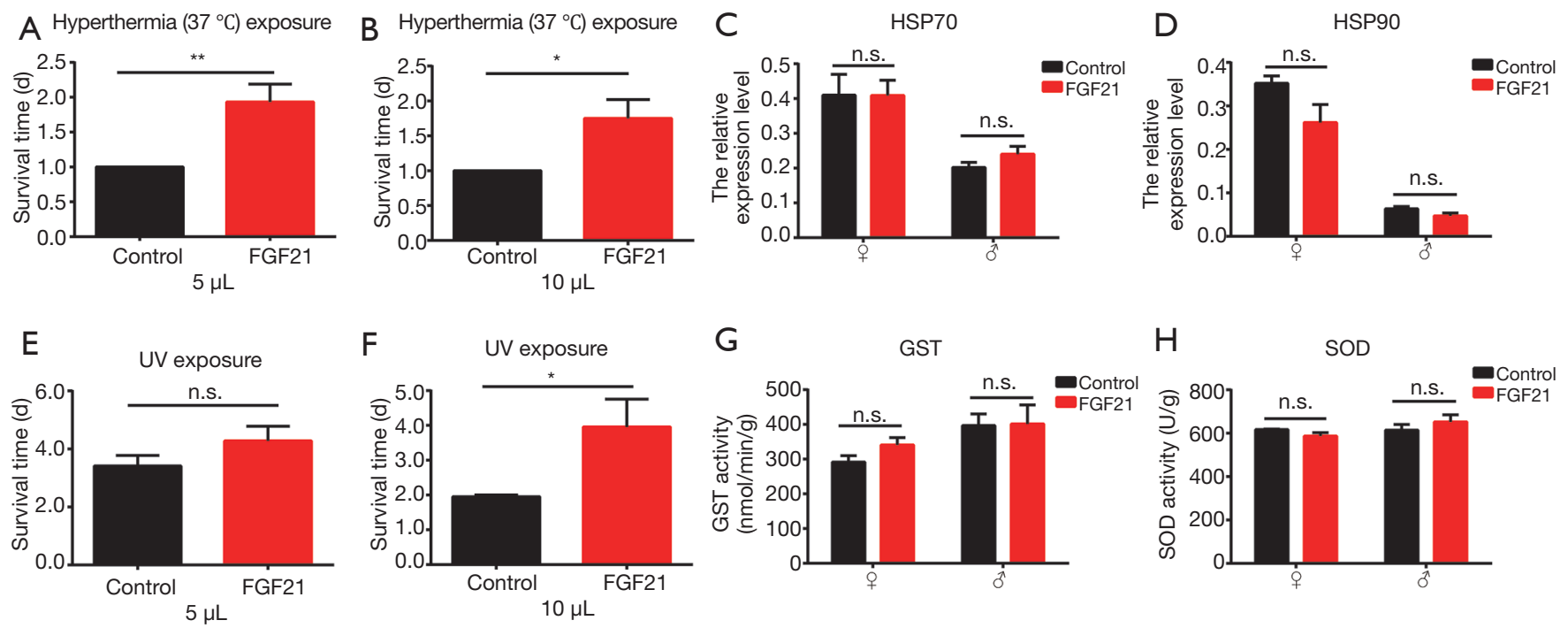

$\mathrm{F}$

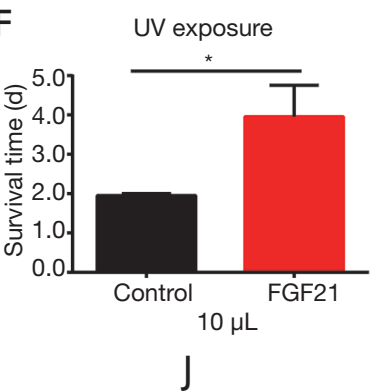

G
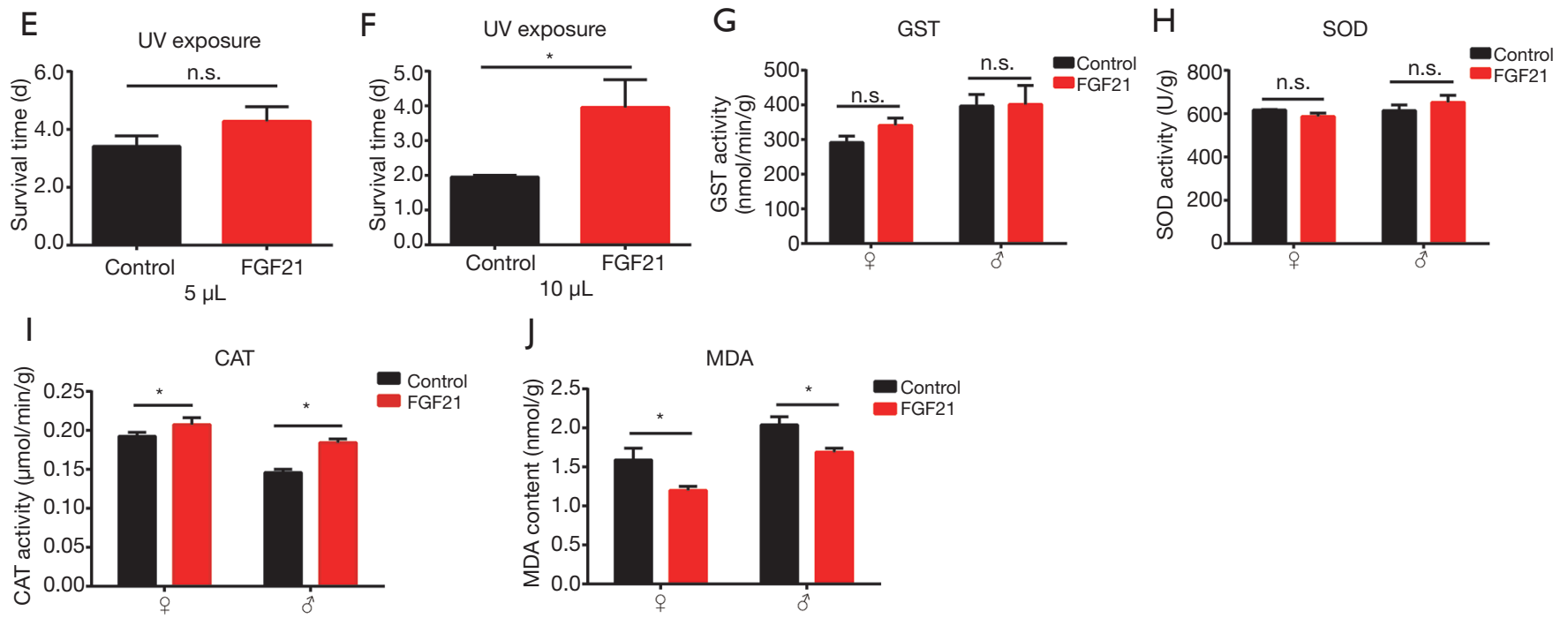

Figure 2 Effects of FGF21 on stress resistance. (A,B) The survival times of moths under hyperthermia $\left(37^{\circ} \mathrm{C}\right)$ exposure after FGF 21 injected: (A) $5 \mu \mathrm{L} 25 \mathrm{ng} / \mu \mathrm{L}$ FGF21 injected (n=15); (B) $10 \mu \mathrm{L} 25 \mathrm{ng} / \mu \mathrm{L}$ FGF21 injected (n=15). (C,D) The expression level of HSP70 and HSP90: (C) HSP70; (D) HSP90. (E,F) The survival times of moths under UV exposure after injection of $25 \mathrm{ng} / \mu \mathrm{L}$ FGF21: (E) $5 \mu \mathrm{L}$ of $25 \mathrm{ng} / \mu \mathrm{L}$ FGF2 1 (treatment group) and $5 \mu \mathrm{L}$ of sterile $\mathrm{H}_{2} \mathrm{O}$ (control) (n=15); (F) $10 \mu \mathrm{L}$ of $25 \mathrm{ng} / \mu \mathrm{L}$ FGF2 (treatment group) and $10 \mu \mathrm{L}$ of sterile $\mathrm{H}_{2} \mathrm{O}$ (control) $(\mathrm{n}=15)$. (G,H,I,J) The detection of enzyme activity and oxidation product content after $\mathrm{UV}$ exposure: $(\mathrm{G})$ the activity of GST; (H) the activity of SOD; (I) the activity of CAT; (J) the content of MDA. Error bars and symbols depict the mean \pm SEM. * $\mathrm{P}<0.05$; **, $\mathrm{P}<0.01$. FGF21, fibroblast growth factor 21; UV, ultraviolet; GST, glutathione-S-transferase; SOD, superoxide dismutase; CAT, catalase; MDA, malondialdehyde; n.s., no significance; SEM, standard error of the mean.

counterpart (Figure $2 I, 7$ ). These results suggest that FGF21-induced improvement of antioxidative capability plays an active role in improving the survival rate under environmental stresses.

\section{FGF21 enhances silkworm antioxidative properties}

FGF21 functions as a stress hormone that activates the organism's defense systems and nonspecifically improves stress tolerance, thereby enhancing protection against aging via suppression of stress $(20,21)$. Therefore, we tested whether the major antioxidant defense systems might be upregulated or the content of the oxidative products might be decreased by FGF21 in silkworms. Several pathological studies have indicated that the GST and SOD activity, and MDA content are oxidative stress biomarkers that can be used to determine the status of stress from an oxidative viewpoint (6). Therefore, GST and SOD activity and MDA content are used as the primary indicators of antioxidation ability. We detected these indicators on day 1 after treatment of silkmoths with FGF21 or controls treatment. The GST and SOD activity were greater in the treatment groups than the control at day 1 (Figure $3 A, B$ ). Meanwhile, the MDA content significantly decreased (Figure $3 C$ ). Previous researches have also reported that FoxO could be activated directly by deacetylation and dephosphorylation alternatively in response to oxidative stress which, in turn, promotes their specificity target genes in antioxidase system 

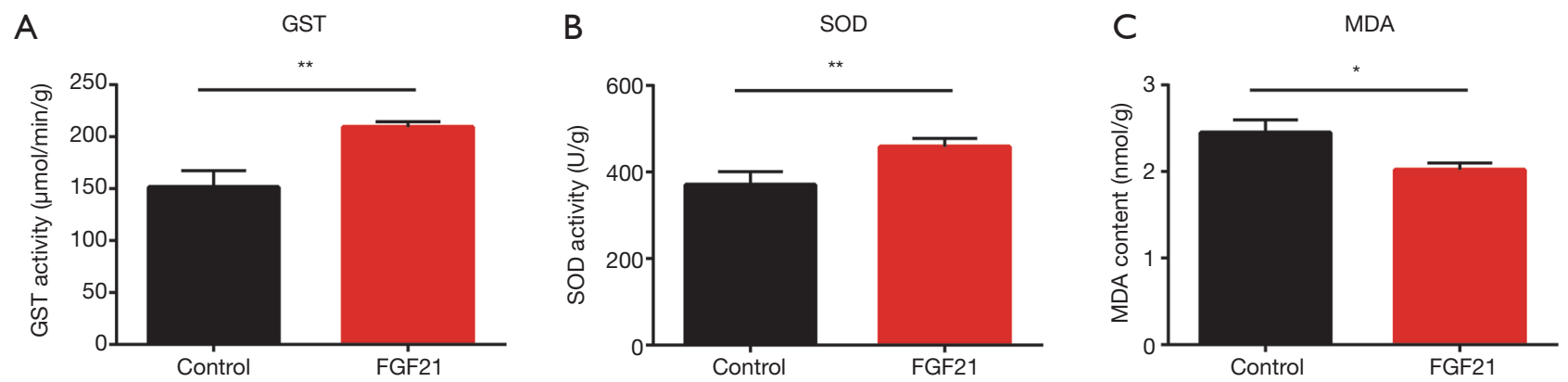

Figure 3 The detection of antioxidant activity in silkworms. (A) GST activity; (B) SOD activity; (C) MDA content. Error bars depict the mean \pm SEM, $\mathrm{n}=8$. *, $\mathrm{P}<0.05 ;{ }^{* *}, \mathrm{P}<0.01$. SEM, standard error of the mean; GST, glutathione-S-transferase; SOD, superoxide dismutase; MDA, malondialdehyde.

including GST and SOD towards stress resistance and then reduce the oxidation product content including MDA $(9,22)$. These results suggest that FGF21 may improve the antioxidative properties in silkworms.

\section{FGF21-induced lifespan extension may be increased via activated AMPK and FoxO}

The FGF receptor assembles a specific receptor complex with the klotho protein and subsequently activates several downstream signaling pathways in a context-dependent manner (23-27). FGF21 is a regulator that alleviates many age-related metabolic and stress disorders. Herein, to detect whether AMPK participates in lifespan elongation in silkworms and to further investigate the underlying signaling network through which FGF21 induces lifespan extension, we detected the expression levels of $B m A K T$, BmAMPK, BmFoxO, BmTor1, and BmTor2 in FGF21injected silkworms and controls.

There were no significant differences in the BmAKT relative expression level after both 24 and $48 \mathrm{~h}$ of treatment and in the control (Figure $4 A$ ). The expression level of $B m A M P K$ was significantly higher at $48 \mathrm{~h}$ than that in the control, but no significant differences were observed at $24 \mathrm{~h}$ (Figure 4B). The relative expression level of BmTor1 and BmTor 2 significantly increased at $48 \mathrm{~h}$ after injection FGF21, whereas no significant differences were found at $24 \mathrm{~h}$ (Figure 4C,D). Expression of $B m F o x O$, a major antistress regulator in cells and organisms, significantly increased at $24 \mathrm{~h}$ (Figure 4E). Many studies have indicated that increases in AMPK and FoxO expression promote longevity (9). Our results indicate that FGF21-induced lifespan extension may be increased by elevated BmAMPK and BmFoxO expression levels.

\section{FGF21 is linked to the sirtuin-coupled longevity pathway}

FGF21 delays endothelial replicative senescence and protects cells from premature senescence through Sirt1 (28). Increased sirtuin activity is also associated with longevity $(9,29,30)$. To detect whether sirtuin takes part in the process of lifespan elongation of silkworm and further excavate the underlying signaling network for FGF21-inducing lifespan extension. However, only five genes (BmSirt2, BmSirt4, BmSirt5, BmSirt6, and BmSirt7) have been identified to belong to the sirtuin family in silkworms, in which Sirt1 does not exist. Therefore, to detect the family member replacing Sirt1 in FGF21 induced longevity in silkworms, we investigated the expression levels of five sirtuin genes in silkworms with or without FGF21 replenishment. The BmSirt2, BmSirt4, and BmSirt6 expression levels were significantly higher after $48 \mathrm{~h}$ in treatment than those in the control group, whereas no significant differences were found at $24 \mathrm{~h}$ (Figure $5 A, B, C$ ). There were no significant differences between the BmSirt5 and BmSirt7 expression levels after 24 and $48 \mathrm{~h}$ of treatment and in the control group (Figure 5D,E). We speculate that BmSirt2/BmSirt4/ BmSirt6 may functionally complement Sirt1 in the regulatory process through which FGF21 activates Sirt1 and thus plays a role in promoting longevity.

\section{Discussion}

The development of potential antiaging drugs is an interesting scientific research field. In this area, mammalian animal models such as mice, rats, rabbits, and monkeys, 
A

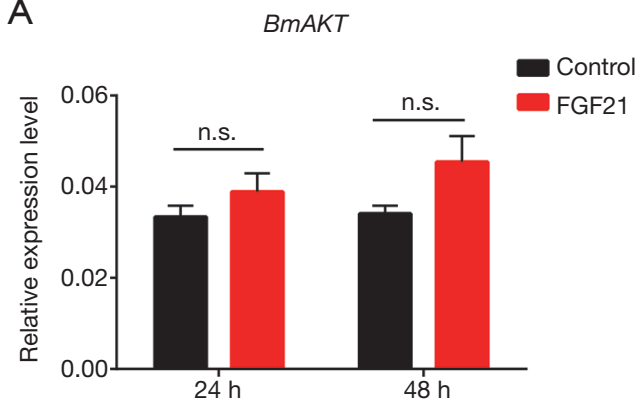

C
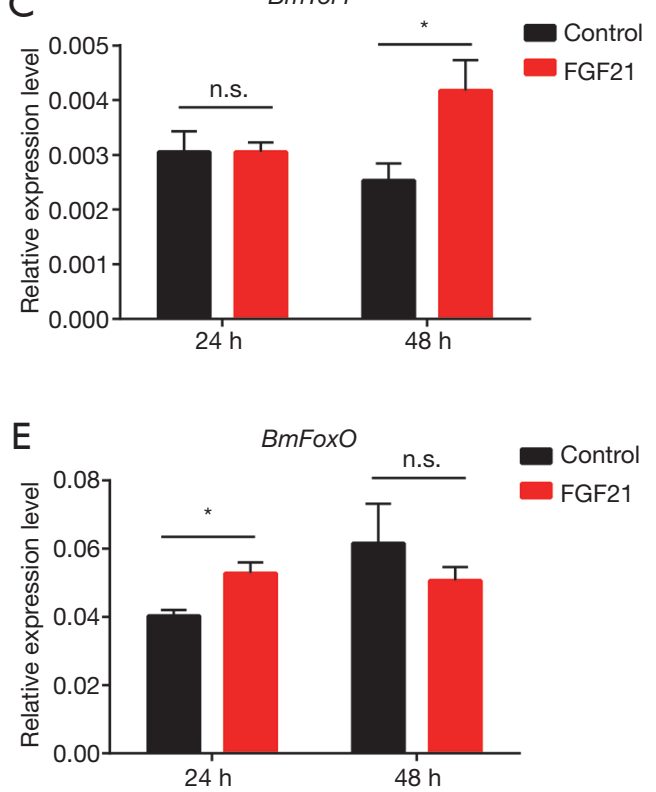

B
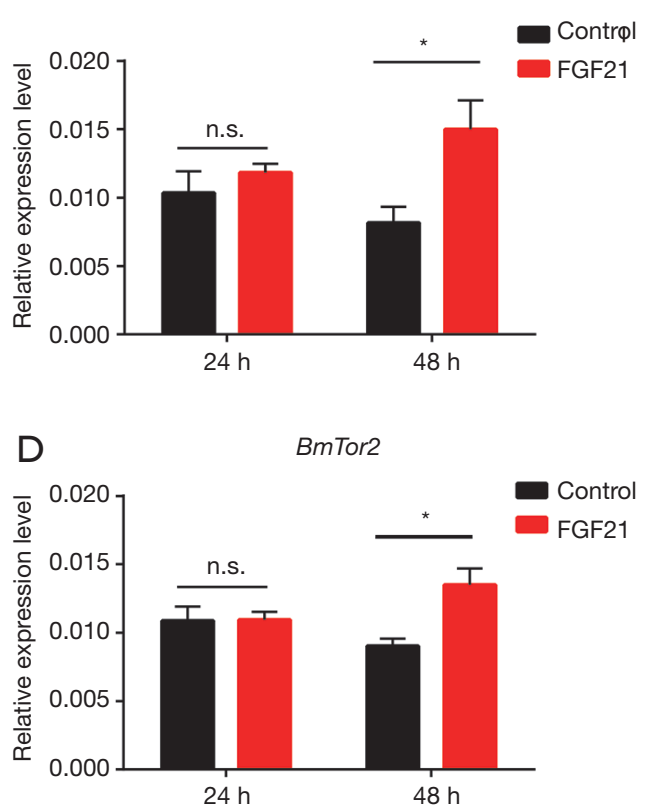

Figure 4 The expression level of FGF21 induced genes at 24 and $48 \mathrm{~h}$ in silkmoths. (A) BmAKT expression level; (B) BmAMPK expression level; (C,D) BmTor1 and BmTor2 expression level; (E) BmFoxO expression level. Error bars depict the mean \pm SEM, $\mathrm{n}=9$. * $\mathrm{P}<0.05$. FGF21, fibroblast growth factor 21 ; n.s., no significance; SEM, standard error of the mean.

has been used for the development of clinical drugs in vivo. However, several problems have been inevitable in the process, particularly, long life cycle, high costs, and ethical issues. Consequently, specific knowledge regarding adequate preclinical invertebrate models is needed. Previous studies have demonstrated that the silkworm is a useful in vivo model as a primary screening system for the discovery and evaluation of anti-infectious drugs, new antibiotics, and anti-diabetes drugs (31-33). This study and our previous studies focused on using the silkworm as an invertebrate animal model system to evaluate the effects of compounds and to provide the new insights into lifespan extension.

In this study, different effects on lifespan were observed between sexes: a significant extension was observed in female, whereas the lifespan was not significantly extended in males. In FGF21 transgenic mice, the high levels of this starvation hormone suppress the activity of growth hormone and decrease the production of IGF, thereby preventing growth and leading to lifespan extension (34). However, the longevity effect has also shown sex differences in mice, in which a greater degree of extension is observed in female (34). In addition, a previous study has suggested that sex differences in response to dietary restriction might possibly result from differences in FGF21 and altered expression of its receptors (35). On the basis of the studies above, we speculate that a dose effect of FGF21 replenishment may exist between the two sexes in terms of lifespan extension. 
A
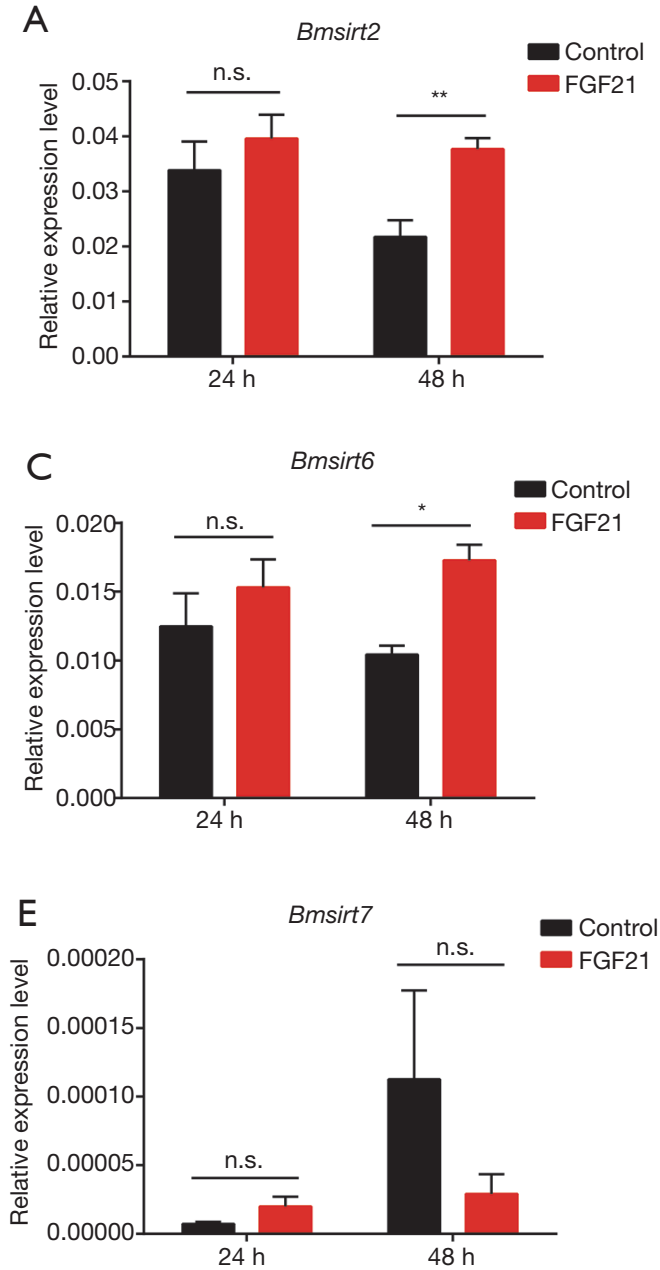

B

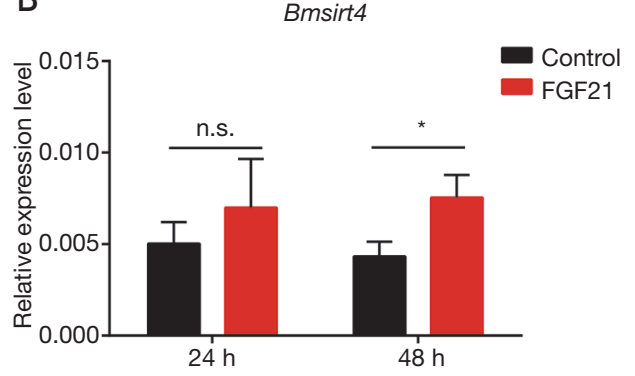

D Bmsirt5

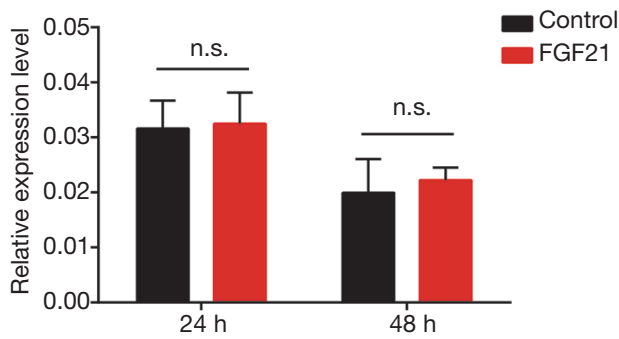

Figure 5 Expression levels of sirtuin genes in FGF21 replenished silkworms. (A) BmSirt2; (B) BmSirt4; (C) BmSirt6; (D) BmSirt5; (E) BmSirt7. Error bars depict the mean $\pm \mathrm{SEM}, \mathrm{n}=9$. * $\mathrm{P}<0.05$; ** $\mathrm{P}<0.01$. FGF21, fibroblast growth factor 21; n.s., no significance; SEM, standard error of the mean.

Oxidative stress is a common environmental stress regarded as the major risk factor for aging. Moreover, FoxO is an important transcriptional factor that regulates the stress response, thereby leading to lifespan extension (36). Previous studies have demonstrated that FoxO promotes oxidative stress resistance in different organisms (37-39). Furthermore, AMPK is a principal energy sensor that plays a central role in maintaining energy balance and tissue integrity (40). Previous studies have revealed that activated AMPK can ultimately upregulate Sirt1 gene expression by enhancing the capacity of nicotinamide phosphoribosyl transferase (NAMPT), an important rate-limiting enzyme in the transformation of nicotinamide (NAM) to nicotinamide adenosine dinucleotide $\left(\mathrm{NAD}^{+}\right)$(41). Consequently, we speculated that FoxO and AMPK may trigger the increase in oxidative stress resistance and may participate in the biological process through which FGF21 replenishment induces lifespan extension in silkworms.

Sirtuin has been identified to be involved in the regulation of metabolic status and stress tolerance under nutritional and environmental stimuli, as well as the regulation of various biological effects that play an important role in the aging process (42). The sirtuin family is classified into seven sirtuin genes (Sirt1-Sirt7). Compared with Drosophila melanogaster, Apis mellifera, and Tribolium castaneum, Bombyx mori has a natural Sirt1 gene deletion. Although all sirtuins include a conserved core structure containing the catalytic domain, the subcellular localization of sirtuin proteins varies (43). The four proteins Sirt1, Sirt3, Sirt6, and Sirt7 are 
normally distributed in the nucleus with different nuclear localization, although this localization is not absolute. Sirt1 plays an important role in the nucleus as well as the cytoplasm $(44,45)$, and Sirt2 is normally present in the cytoplasm and can be bound to chromatin in mitosis (46).

The FGF and FGF receptor (FGFR) have orthologous homologous in silkworm. One critical feature of transcriptional activation of endocrine FGF21 is that they require the Klotho gene encoding transmembrane proteins as coreceptors for binding their cognate FGF21 receptors and exerting their biological activities (47). FGF21 could been transferred into intracellular through the $\beta$ KlothoFGFR channel on the cytomembrane. These genes have explicit orthologous homologous genes in silkworm. So, we speculate that there is an intracellular signaling pathway for FGF21. FGF21 is a regulator that can alleviate many age-related metabolic and stress disorders, through the mechanisms including the activation of AMPK and the Sirt1 signaling pathway (5). We found that FoxO may respond to AMPK activation by FGF21. Silkworms with intrinsic Sirt1 deletion may aid in identification of the Sirt1 functional replacer. Through silkworm characterization, we detected the paralogous genes of BmSirt1 in silkworms and found that the expression levels of BmSirt2, BmSirt4, and BmSirt6 increased in FGF21 replenished silkworms. Given the notably consistent expression profile between $B m A M P K$ and BmSirt2/BmSirt4/BmSirt6 in silkworms, we speculate that BmSirt2/BmSirt4/BmSirt6 may at least partially compensate for the absence of Sirt1.

\section{Acknowledgments}

We thank doctoral candidates Jianfei Zhang, Zheng Li, and Master degree candidate Lian Liu for valuable support in software usage and providing suggestions on research material preparation. We also acknowledge the support of the National Natural Science Foundation of China [No. 31472153]. We thank International Science Editing (http://www.internationalscienceediting.com) for editing this manuscript.

Funding: This work was supported by the National Natural Science Foundation of China (grant No. 31830094 , 31902215), the Hi-Tech Research and Development 863 Program of China Grant (grant No. 2013AA102507), the Fundamental Research Funds for the Central Universities in China (grant No. XDJK2019C014), project funded by the Chongqing Special Postdoctoral Science Foundation (grant No. XmT2018058); and Funds of the China
Agriculture Research System (grant No. CARS-18ZJ0102).

\section{Footnote}

Conflicts of Interest: The authors have no conflicts of interest to declare.

Ethical Statement: The authors are accountable for all aspects of the work in ensuring that questions related to the accuracy or integrity of any part of the work are appropriately investigated and resolved. All of the above animal procedures are consistent with the statement of Southwest University on the Welfare of Animals.

Open Access Statement: This is an Open Access article distributed in accordance with the Creative Commons Attribution-NonCommercial-NoDerivs 4.0 International License (CC BY-NC-ND 4.0), which permits the noncommercial replication and distribution of the article with the strict proviso that no changes or edits are made and the original work is properly cited (including links to both the formal publication through the relevant DOI and the license). See: https://creativecommons.org/licenses/by-nc-nd/4.0/.

\section{References}

1. Peifley KA, Alberts GF, Hsu DK, et al. Heparin-binding epidermal growth factor-like growth factor regulates fibroblast growth factor-2 expression in aortic smooth muscle cells. Circ Res 1996;79:263-70.

2. Brachmann CB, Sherman JM, Devine SE, et al. The SIR2 gene family, conserved from bacteria to humans, functions in silencing, cell cycle progression, and chromosome stability. Genes Dev 1995;9:2888-902.

3. Itoh N, Ornitz DM. Functional evolutionary history of the mouse Fgf gene family. Dev Dyn 2008;237:18-27.

4. Salminen A, Kauppinen A, Kai K. FGF21 activates AMPK signaling: impact on metabolic regulation and the aging process. J Mol Med (Berl) 2017;95:123-31.

5. Salminen A, Kaarniranta K, Kauppinen A. Integrated stress response stimulates FGF21 expression: Systemic enhancer of longevity. Cell Signal 2017;40:10-21.

6. Salminen A, Kaarniranta K, Kauppinen A. Regulation of longevity by FGF21: Interaction between energy metabolism and stress responses. Ageing Res Rev 2017;37:79-93.

7. Yu Y, He J, Li S, et al. Fibroblast growth factor 21 (FGF21) 


\section{Page 10 of 11}

inhibits macrophage-mediated inflammation by activating $\mathrm{Nrf2}$ and suppressing the NF- $\mathrm{\kappa B}$ signaling pathway. Int Immunopharmacol 2016;38:144-52.

8. Deepashree S, Niveditha S, Shivanandappa T, et al. Oxidative stress resistance as a factor in aging: evidence from an extended longevity phenotype of Drosophila melanogaster. Biogerontology 2019;20:497-513.

9. Kenyon CJ. The genetics of ageing. Nature 2010;464:504-12.

10. Chen C, Song JB, Chen M, et al. Rhodiola rosea extends lifespan and improves stress tolerance in silkworm, Bombyx mori. Biogerontology 2016;17:373-81.

11. Song J, Jiang G, Zhang J, et al. Metformin prolongs lifespan through remodeling the energy distribution strategy in silkworm, Bombyx mori. Aging (Albany NY) 2019;11:240-8.

12. Song J, Zhang J, Dai F. Advantages and Limitations of Silkworm as an Invertebrate Model in Aging and Lifespan Research. OAJ Gerontol \& Geriatric Med 2018;4:555641.

13. Song J, Chen M, Li Z, et al. Astragalus polysaccharide extends lifespan via mitigating endoplasmic reticulum stress in the silkworm, Bombyx mori. Aging Dis 2019;10:1187-98.

14. Wang GH, Xia QY, Cheng DJ, et al. Reference genes identified in the silkworm Bombyx mori during metamorphism based on oligonucleotide microarray and confirmed by qRT-PCR. Insect Sci 2010;15:405-13.

15. Longo VD, Fabrizio P. Visions \& Reflections? Regulation of longevity and stress resistance: a molecular strategy conserved from yeast to humans? Cell Mol Life Sci 2002;59:903-8.

16. Zhou KI, Pincus Z, Slack FJ. Longevity and stress in Caenorhabditis elegans. Aging 2011;3:733-53.

17. Epel ES, Lithgow GJ. Stress biology and aging mechanisms: toward understanding the deep connection between adaptation to stress and longevity. J Gerontol A Biol Sci Med Sci 2014;69 Suppl 1:S10-6.

18. Fazeli PK, Lun M, Kim SM, et al. FGF21 and the late adaptive response to starvation in humans. J Clin Invest 2015;125:4601-11.

19. Kim SH, Kim KH, Kim HK, et al. Fibroblast growth factor 21 participates in adaptation to endoplasmic reticulum stress and attenuates obesity-induced hepatic metabolic stress. Diabetologia 2015;58:809-18.

20. Wiegant FAC, Surinova S, Ytsma E, et al. Plant adaptogens increase lifespan and stress resistance in C. elegans. Biogerontology 2009;10:27-42.

21. Kim KH, Lee MS. FGF21 as a stress hormone: the roles of
Song et al. FGF21 elongates lifespan and improves stress tolerance

FGF21 in stress adaptation and the treatment of metabolic diseases. Diabetes Metab J 2014;38:245-51.

22. Kenyon C. The plasticity of aging: insights from longlived mutants. Cell 2005;120:449-60.

23. Chau MD, Gao J, Yang Q, et al. Fibroblast growth factor 21 regulates energy metabolism by activating the AMPKSIRT1-PGC-1alpha pathway. Proc Natl Acad Sci U S A 2010;107:12553-8.

24. Patel V, Adya R, Chen J, et al. Novel insights into the cardio-protective effects of FGF21 in lean and obese rat hearts. PLoS One 2014;9:e87102.

25. Zhang C, Huang Z, Gu J, et al. Fibroblast growth factor 21 protects the heart from apoptosis in a diabetic mouse model via extracellular signal-regulated kinase 1/2-dependent signalling pathway. Diabetologia 2015;58:1937-48.

26. Fisher FM, Maratosflier E. Understanding the Physiology of FGF21. Annu Rev Physiol 2016;78:223-41.

27. Woo YC, Xu A, Wang Y, et al. Fibroblast growth factor 21 as an emerging metabolic regulator: clinical perspectives. Clin Endocrinol (Oxf) 2013;78:489-96.

28. Yan J, Wang J, Huang H, et al. Fibroblast growth factor 21 delayed endothelial replicative senescence and protected cells from $\mathrm{H} 2 \mathrm{O} 2$-induced premature senescence through SIRT1. Am J Transl Res 2017;9:4492-501.

29. Bishop NA, Leonard G. Genetic links between diet and lifespan: shared mechanisms from yeast to humans. Nat Rev Genet 2007;8:835-44.

30. Zhang Y, Xie Y, Berglund ED, et al. The starvation hormone, fibroblast growth factor-21, extends lifespan in mice. Elife 2012;1:e0065.

31. Uchida R, Iwatsuki M, Kim YP, et al. Nosokomycins, new antibiotics discovered in an in vivo-mimic infection model using silkworm larvae. I: Fermentation, isolation and biological properties. J Antibiot (Tokyo) 2010;63:151-5.

32. Kaito C, Akimitsu N, Watanabe H, et al. Silkworm larvae as an animal model of bacterial infection pathogenic to humans. Microb Pathog 2002;32:183-90.

33. Matsumoto Y, Sumiya E, Sugita T, et al. An invertebrate hyperglycemic model for the identification of anti-diabetic drugs. Plos One 2011;6:e18292.

34. Zhang Y, Xie Y, Berglund ED, et al. The starvation hormone, fibroblast growth factor-21, extends lifespan in mice. eLife 2012;1:e0065.

35. Larson KR, Russo KA, Fang Y, et al. Sex Differences in the Hormonal and Metabolic Response to Dietary Protein Dilution. Endocrinology 2017;158:3477-87.

36. Mithoefer K, McAdams T, Williams RJ, et al. Clinical 
efficacy of the microfracture technique for articular cartilage repair in the knee: an evidence-based systematic analysis. Am J Sports Med 2009;37:2053-63.

37. Kops GJPL, Dansen TB, Polderman PE, et al. Forkhead transcription factor FOXO3a protects quiescent cells from oxidative stress. Nature 2002;419:316-21.

38. Essers MA, de Vries-Smits LM, Barker N, et al. Functional interaction between beta-catenin and FOXO in oxidative stress signaling. Science 2005;308:1181-4.

39. Akasaki Y, Alvarez-Garcia O, Saito M, et al. FoxO transcription factors support oxidative stress resistance in human chondrocytes. Arthritis Rheumatol 2014;66:3349-58.

40. Fang J, Yang J, Wu X, et al. Metformin alleviates human cellular aging by upregulating the endoplasmic reticulum glutathione peroxidase 7. Aging Cell 2018;17:e12765.

41. Wang LF, Wang XN, Huang CC, et al. Inhibition of NAMPT aggravates high fat diet-induced hepatic steatosis in mice through regulating Sirt1/AMPK $\alpha /$ SREBP1

Cite this article as: Song JB, Hao KG, Chen X, Zhang YH, Cheng ZL, Mao S, Tang YX, Tong XL, Dai FY. Fibroblast growth factor 21 prolongs lifespan and improves stress tolerance in the silkworm, Bombyx mori. Ann Transl Med 2020;8(5):220. doi: 10.21037/atm.2020.01.18 signaling pathway. Lipids Health Dis 2017;16:82.

42. Finkel T, Deng CX, Mostoslavsky R. Recent progress in the biology and physiology of sirtuins. Nature 2009;460:587-91.

43. Cantó C, Auwerx J. Caloric restriction, SIRT1 and longevity. Trends Endocrinol Metab 2009;20:325-31.

44. Michishita E, Park JY, Burneskis JM, et al. Evolutionarily conserved and nonconserved cellular localizations and functions of human SIRT proteins. Mol Biol Cell 2005;16:4623-35.

45. Tanno M, Sakamoto J, Miura T, et al. Nucleocytoplasmic shuttling of the NAD+-dependent histone deacetylase SIRT1. J Biol Chem 2007;282:6823-32.

46. Mostoslavsky R, Chua KF, Lombard DB, et al. Genomic instability and aging-like phenotype in the absence of mammalian SIRT6. Cell 2006;124:315-29.

47. Kuro-o M. Klotho and $\beta$ Klotho. In: Kuro-o M. editor. Endocrine FGFs and Klothos. New York, NY: Springer US, 2012:25-40. 